\title{
The A242T Mutation in the Low-density Lipoprotein Receptor-related Protein 5 Gene in One Chinese Family with Osteosclerosis
}

\author{
Chun Wang ${ }^{1}$, Bao-Hong Zhang ${ }^{2}$, Hao Zhang ${ }^{1}$, Jin-Wei He${ }^{1}$, Yun-Qiu $\mathrm{Hu}^{1}$, Miao $\mathrm{Li}^{1}$, \\ Hua Yue ${ }^{1}$, Wei-Wei Hu ${ }^{1}$, Jie-Mei Gu ${ }^{1}$, Yu-Juan Liu ${ }^{1}$, Wen-Zhen Fu ${ }^{1}$ and Zhen-Lin Zhang ${ }^{1}$
}

\begin{abstract}
Objective Osteosclerosis (OMIM: 144750) is a type of autosomal dominant bone disease caused by a mutation in the low-density lipoprotein receptor-related protein 5 (LRP5) gene. The case of a Chinese family with two affected individuals is reported in the present study in order to investigate the clinical characteristics and virulence genes of this sclerosing bone disorder.

Methods Biochemical and radiographic examinations and bone mineral density (BMD) and genetic analyses were performed in two patients and eight other family members.

Results The 40-year-old proband (II-1) and her 64-year-old mother (I-2) both had chronic lumbodorsal pain, an elongated mandible and torus palatinus in the center of the hard palate. No fractures were observed in any of the family members. Skull, mandibular and pelvic X-rays in each of the two patients revealed thickened cranial plates, an enlarged sella turcica, an elongated mandible and cortical thickening of the long bones. The BMD values of the two patients was significantly higher than the standard age- and sex-matched adult mean reference values. Both patients had higher serum sclerostin levels, while their renal function markers and serum calcium, phosphonium, parathyroid hormone $(\mathrm{PTH})$ and 25(OH)D levels were within the normal ranges. The heterozygous missense mutation p.Ala242Thr in exon 4 of the LRP5 gene was detected in the two patients, while the other family members and 200 healthy donors had normal wild-type genotypes. Conclusion The A242T mutation in the LRP5 gene resulted in a high bone mass phenotype with an elongated mandible and torus palatinus in this osteosclerotic family.
\end{abstract}

Key words: osteosclerosis, $L R P 5$, mutation, sclerostin

(Intern Med 52: 187-192, 2013)

(DOI: 10.2169/internalmedicine.52.8164)

\section{Introduction}

Osteosclerosis (OMIM: 144750) is a type of autosomal dominant bone disease first described by Worth and Wollin in 1966 (1). The main clinical characteristics of this disease include a widened and deepened mandible with an increased gonial angle, endosteal sclerosis of the calvaria with a loss of the diploe, osteosclerosis and hyperostosis of the mandible with an absence of the normal antegonial notches, endosteal sclerosis of the diaphyses of the long bones (includ- ing the metacarpals and metatarsals) and osteosclerosis of the pelvis. Torus palatinus in the hard palate is observed in approximately $25 \%$ of female patients (2) and can lead to malocclusion or tooth loss. Notably, almost no fractures occur in affected patients. The course of osteosclerosis is benign and osteosclerotic patients exhibit normal height, proportions, intelligence and longevity (3).

In 2002, the disease-causing gene for high bone density with a thickened mandible and torus palatinus was localized on low-density lipoprotein receptor-related protein 5 (LRP5) (4). The LRP5 gene on chromosome 11q13, which

${ }^{1}$ Metabolic Bone Disease and Genetics Research Unit, Department of Osteoporosis and Bone Diseases, Shanghai Sixth People's Hospital affiliated with Shanghai Jiao Tong University, China and ${ }^{2}$ Capital Institute of Pediatrics, China

Received for publication May 16, 2012; Accepted for publication July 17, 2012

Correspondence to Dr. Zhen-Lin Zhang, zzl2002@medmail.com.cn 


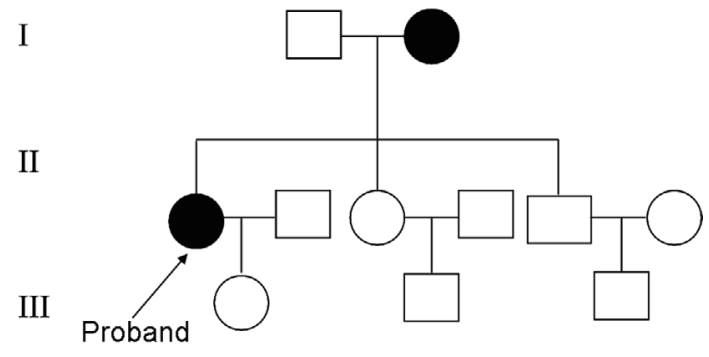

Figure 1. The pedigree tree of one Chinese Han ethnic family with osteosclerosis.

codes for co-receptors in the canonical Wnt signaling pathway, has been shown to play an important role in skeletal homeostasis and to be involved in the pathogenesis of several bone disorders. LRP5 mutations are of two general types: (1) autosomal dominant gain-of-function missense mutations resulting in a high bone mass phenotype and (2) autosomal recessive loss-of-function mutations resulting in a low bone mass phenotype (osteoporosis-pseudoglioma (OPPG) syndrome) (3-5). In 2002, Little (6) and Boyden (4) reported that the G171V mutation is a gain-of-function missense mutation that contributes to high bone density by impairing the action of a normal antagonist of the Wnt pathway and thus increasing Wnt signaling. One year later, six novel missense mutations in the LRP5 gene, T253I, D111Y, G171R, A214T, A214V and A242T, were identified by Van Wesenbeeck (3) as being disease-causing mutations for high bone density.

In the current study, we aimed to characterize the clinical manifestations and molecular defects observed in a Chinese family affected by osteosclerosis.

\section{Materials and Methods}

\section{Study subjects}

Two affected individuals (I-2 and II-1) and eight other family members from a Chinese Han ethnic family were recruited by the Department of Osteoporosis and Bone Diseases outpatient clinic. Their pedigree tree is shown in Fig. 1. Their clinical phenotypes, including clinical symptoms and auditory acuity, and medical histories, including fracture history, family history, developmental history and childbearing history, were collected through questionnaires and medical examinations.

Two hundred age- and sex-matched healthy donors were recruited as controls for a mutation analysis (7).

This study was approved by the Ethics Committee of the Sixth People's Hospital affiliated with Shanghai Jiao Tong University. Written informed consent for this study was obtained from all participants.

\section{Biochemical and radiographic examinations}

A full blood count and the levels of serum calcium (Ca), phosphonium (P), alkaline phosphatase (ALP), blood urea nitrogen (BUN), serum creatinine (Scr), creatine kinase $(\mathrm{CK})$ and its $\mathrm{MB}$ isoenzyme (CKMB), lactate dehydrogenase (LDH), parathyroid hormone (PTH) and 25hydroxyvitamin $\mathrm{D}(25(\mathrm{OH}) \mathrm{D})$ were measured in $\mathrm{I}-2$, II-1 and their family members. The presence of the bone absorption marker $\beta$ cross-linked $C$-telopeptides of type I collagen $(\beta-\mathrm{CTX})$ in serum was determined using an electrochemiluminescent method (Roche Diagnostic, Mannheim, Germany). Moreover, the serum sclerostin level was determined using a quantitative sandwich enzyme-linked immunosorbent assay (ELISA) (Biomedica, Vienna, Austria). The inter-assay coefficient of variability $(\mathrm{CV})$ was $7 \%$. X-ray radiographs of the thoracic and lumbar vertebrae, limbs, hips, mandible and skull were taken in both I-2 and II-1.

\section{Bone mineral density (BMD) measurements}

The BMDs of the anteroposterior lumbar spine 1-4 (L1-4) and the left proximal femur, including the femoral neck and total hip, were measured using a lunar prodigy dual energy X-ray absorptiometry (DXA) densitometer (Lunar Corp, Madison, WI, USA). These data were analyzed using Prodigy enCORE software (ver. 6.70, standard-array mode; GE Healthcare, Madison, WI, USA). The machine was calibrated daily, and the CV values of the DXA measurements obtained from triplicate measurements of the same 15 individuals for L1-4, total hip, femoral neck and trochanter were $1.39 \%, 0.70 \%, 2.22 \%$ and $1.41 \%$, respectively (8). The long-term reproducibility of the DXA data during the trial based on weekly repeated phantom measurements was $99.55 \%$ (9). Height and body weight were measured using standard equipment.

\section{Mutation analysis}

Genomic DNA was isolated from peripheral blood leukocytes using the conventional phenol-chloroform extraction method. The entire coding region and adjacent splice sites of the CLCN7 (GenBank Accession Number RefSeq NG_ 007567.1), LRP5 (GenBank Accession Number RefSeq NG_ 015835.1) and Sclerostin (SOST) (GenBank Accession Number RefSeq NG_008078.2) genes were amplified and sequenced for I-2, II-1 and their family members. Further validation was performed in the 200 healthy donors.

\section{Results}

Clinical and genetic findings in the two affected individuals

The 40-year-old proband (II-1) and her 64-year-old mother (I-2) suffered from severe lumbodorsal pain. II-1 was $172.5 \mathrm{~cm}$ in height and $87.5 \mathrm{~kg}$ in weight, and I-2 was $167.0 \mathrm{~cm}$ in height and $64 \mathrm{~kg}$ in weight. An elongated mandible and torus palatinus in the center of the hard palate were observed in both patients (Fig. 2). Notably, no fractures, anemia, thrombocytopenia, splenomegaly, nerve deafness or visual impairments were reported by any of the family members. 


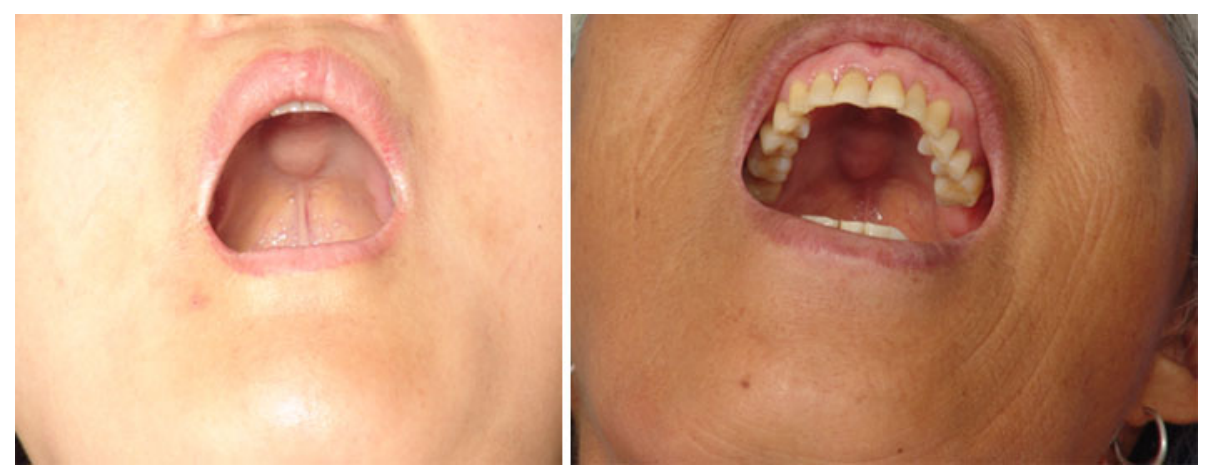

Figure 2. A torus palatinus can be seen in the center of the hard palate in II-1 (left) and I-2 (right).
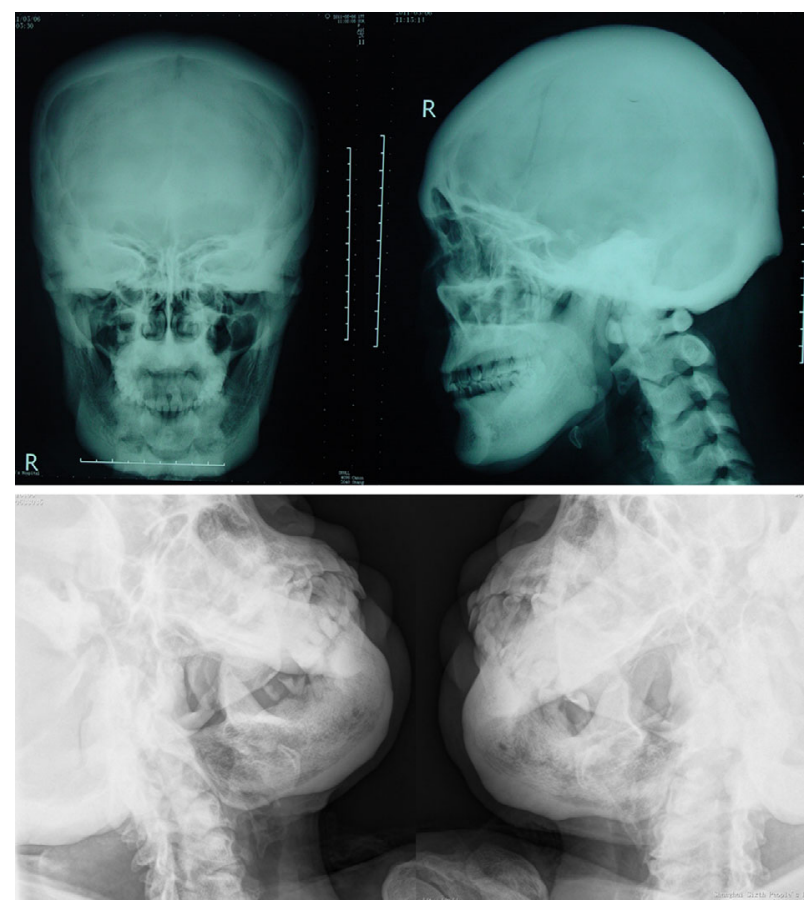

Figure 3. X-rays of the skull and mandible of II-1 (upper) and I-2 (lower) showing osteosclerosis of the skull with an enlarged sella turcica and an elongated mandible.

X-rays of the skull, mandible, humerus, lumber spine and pelvis in the two patients are presented in Figs. 3-5. Typical thickening of the cranial plates, an elongated mandible, cortical thickening of the long bones and degenerative changes were observed in both affected individuals.

The BMD values of the two patients were significantly higher than the standard age- and sex-matched adult mean reference values. The $\mathrm{Z}$ scores of the L1-4, femoral neck, trochanter and total hip in II-1 were 5.5 SD, 7.9 SD, 8.5 SD and 7.6 SD, while the $\mathrm{Z}$ scores for the same four sites in I-2 were 8.1 SD, 5.6 SD, 4.7 SD and 5.0 SD. The family members had normal BMD values compared with the agespecific reference values.

The serum sclerostin levels in II-1 and I-2 were 64.75 $\mathrm{pmol} / \mathrm{L}$ and $60.55 \mathrm{pmol} / \mathrm{L}$, respectively. Because the normal range in the healthy controls $(n=15)$ in our laboratory was 11.08 to $29.01 \mathrm{pmol} / \mathrm{L}$, the values for II- 1 and I-2 were ap-

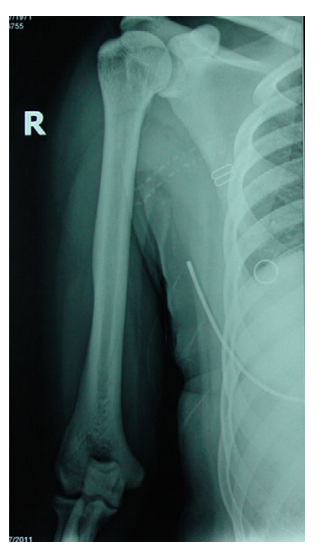

Figure 4. X-ray of the humerus of II-1 showing cortical thickening of the humerus with a normal external shape.

proximately 2.23 and 2.08 times the upper limit of normal (ULN). Elevated serum ALP and $\beta$-CTX levels were observed in patient I-2 only, while the levels of renal function markers, serum calcium and phosphonium, ALP, PTH, 25(OH)D and $\beta$-CTX in patient II-1 and the other family members were mainly within the normal ranges (Table).

The heterozygous missense mutation p.Ala242Thr in exon 4 of LRP5 [dbSNP: rs121908670] was detected in each patient. Both patients harbored the G/A genotype (Fig. 6), while the other family members and the 200 healthy donors had the normal wild-type G/G genotype.

\section{Discussion}

Bone is a dynamic tissue that is regulated by the balanced processes of bone formation and bone resorption. Increased bone formation, decreased bone resorption or imbalanced processes between bone formation and resorption can give rise to skeletal pathologies with increased bone density. Unlike osteoporosis, diseases with a high bone mineral density are usually monogenic genetic disorders. High bone density phenotypes include autosomal dominant osteopetrosis, autosomal dominant osteosclerosis, endosteal hyperostosis and Van Buchem disease.

The major disease-causing gene of osteosclerosis is the LRP5 gene, which contains 23 exons with 1,615 amino ac- 


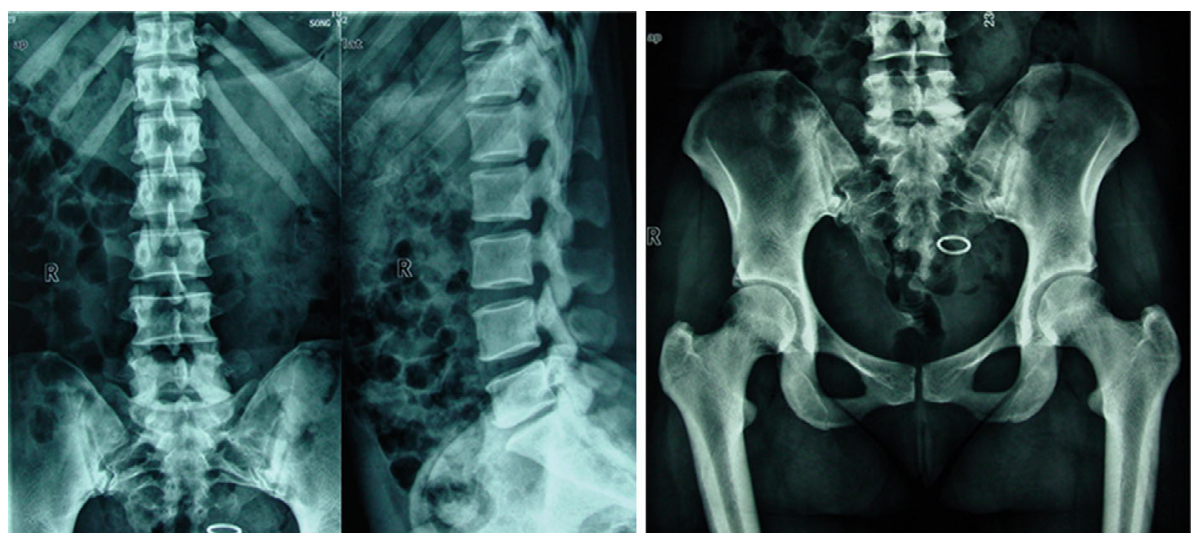

Figure 5. X-rays of the lumber spine and pelvis of II-1 showing diffuse osteosclerosis and degenerative changes without the radiographic signatures of "sandwich vertebrae" or a bone-within-bone appearance in the iliac wings.

Table. The Biochemical Parameters and Bone Turnover Markers of Two Affected Individuals with Osteosclerosis and Their Family Members

\begin{tabular}{|c|c|c|c|c|c|c|c|c|c|}
\hline Subject & $\begin{array}{l}\text { Age } \\
\text { (yr) }\end{array}$ & $\begin{array}{l}\text { ALP } \\
(11-112 \mathrm{U} / \mathrm{L})\end{array}$ & $\begin{array}{l}\mathrm{Ca} \\
(2.08-2.60 \mathrm{mmol} / \mathrm{L})\end{array}$ & $\begin{array}{l}\mathrm{P} \\
(0.80-1.60 \mathrm{mmol} / \mathrm{L})\end{array}$ & $\begin{array}{l}\text { Scr } \\
(53-115 \mu \mathrm{mol} / \mathrm{L})\end{array}$ & $\begin{array}{l}\text { PTH } \\
(15-65 n g / L)\end{array}$ & $\begin{array}{l}25(\mathrm{OH}) \mathrm{D} \\
(11.1-42.9 \mathrm{ng} / \mathrm{mL})\end{array}$ & $\begin{array}{l}\beta-C T X \\
(<650 \text { ng/L) }\end{array}$ & $\begin{array}{l}\text { Sclerostin } \\
(11.08-29.01 \mathrm{pmol} / \mathrm{L})\end{array}$ \\
\hline II-1 & 40 & 60 & 2.39 & 1.24 & 64 & 25.51 & $9.66^{*}$ & 140 & $64.75^{*}$ \\
\hline $\mathrm{I}-2$ & 64 & $115^{*}$ & 2.11 & 1.56 & 54 & 20.35 & 11.33 & $1500^{*}$ & $60.55^{*}$ \\
\hline FM1 & 64 & 96 & 2.21 & 0.96 & 54 & 27.52 & 17.36 & 262 & 13.58 \\
\hline FM2 & 36 & 52 & 2.31 & 1.14 & 59 & 23.74 & $<4.00^{*}$ & 346 & 11.82 \\
\hline FM3 & 31 & 80 & 2.35 & 1.22 & 71 & 29.54 & 14.73 & 623 & $29.98 *$ \\
\hline
\end{tabular}

FM:family member, II-1 was the proband, I-2 was II-1's mother, FM1 was II-1's father, FM2 was II-1's younger sister, and FM was II-1's younger brother. The abnormal parameters were labeled with *.

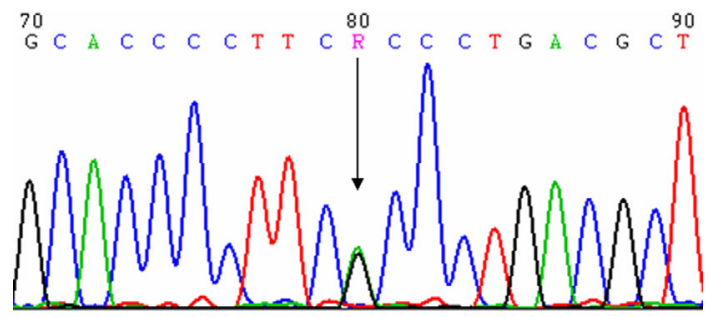

Figure 6. The A242T mutation is a 724G $>$ A transition in exon 4 of the LRP5 gene (arrows).

ids. LRP5 is a type I transmembrane receptor comprised of a long extracellular region (1,376 aa), a single membrane spanning segment (22 aa) and a cytoplasmic region (207 aa) (10). The extracellular domain contains four $\beta$-propellers ( 265 aa each) that alternate with four epidermal growth factor (EGF) receptor-like cysteine-rich repeats $(\sim 42$ aa each). The structural model of the LRP5 first $\beta$-propeller module resembles the Tyrosine, Tryptophan, Threonine and Aspartate (YWTD)/EGF domain pair of low-density lipoprotein receptors (LDLRs) and consists of six blades or motifs ( 43 aa) (11). The LRP5 gene has been shown to be involved in both osteoporosis-pseudoglioma syndrome and the high bone mass phenotype and is an important regulator of peak bone mass in vertebrates $(12,13)$.

In 2002, Kato (14) demonstrated that LRP5 is expressed in the osteoblasts lining the endosteal and trabecular bone surfaces. Mutated LRP5 proteins likely exhibit reduced affinity to and inhibition by Dickkopf1 (DKK1). The altered affinity of LRP5 receptors to specific Wnt ligands might lead to the development of the phenotype of high bone mass $(15,16)$. A substantial amount of evidence has shown that LRP5 variants activate Wnt signaling by relieving Dkk 1-mediated inhibition of $\beta$-catenin/TCF signaling $(3,15,17,18)$. In 2007, Bhat and colleagues (19) demonstrated that the first $\beta$-propeller in LRP5 is important for Dkk1 functioning and Wnt signaling. It plays a potential functional role through intramolecular interactions with the Dkk1 binding domain of LRP5. In 2003, the identified gainof-function mutations for high bone density in the LRP5 gene, such as T253I, G171V, G171R, D111Y, A214T, A214V and A242T, were reviewed by Van Wesenbeeck (3). Investigators have demonstrated that all of the mutations are located in the amino terminal part of the gene in exons 2, 3 and 4 where encoding takes place for the first of four propellers of the LRP5 protein before the first EGF-like domain.

In the present study, the heterozygous A242T mutation was detected in both I-1 and II-2. This mutation is located in the first $\beta$-propeller module, which is very important for Dkk1 functioning and Wnt signaling (19). The clinical manifestations of this hot spot mutation in four families from Portland, France and Sardinia have been previously summarized by Van Wesenbeeck (3). Among these four 
families, the patients from Portland with endosteal hyperostosis each exhibit cortical thickening of the long bones with no alterations in external shape, remarkable resistance of the bones to fractures, an elongated mandible, a decreased gonial angle, torus palatinus and increased BMD in the calvarium, mandible and endosteal surface of the long bones $(20,21)$. The A242T mutation is also present in a previously described Sardinian family with at least five members affected with van Buchem disease (22), all of whom have osteosclerosis of the skull and the cortices of the long bones and an enlarged mandible. In another French family, two patients with osteopetrosis each have osteomyelitis of the jaw, hearing problems due to small auditory canals, diffuse osteosclerosis of the trabecular and cortical bones and osteosclerosis of the skull with an enlargement of the cranial vault.

Consistent with previous studies, the typical clinical manifestations of the two patients in the present study included an elongated mandible and torus palatinus in the center of the hard palate. X-rays of each patient showed cortical thickening of the long bones, osteosclerosis of the skull with an enlarged sella turcica and an elongated mandible. No typical radiographic signatures of osteopetrosis autosomal dominant type 2 (OPTA2) such as dense bands of sclerosis parallel to the vertebral endplates ("sandwich vertebrae" appearance) or bone-within-bone in the iliac wings were observed. Elevated levels of the bone resorption marker $(\beta$ CTX) and the bone formation marker (ALP) were observed in patient I-2. This finding may be due to an imbalance between the gain-of-function A242T missense mutation and the high bone turnover observed in postmenopausal women. The two affected individuals and their family members were either 25OHD insufficient ( $<$ or $=29 \mathrm{ng} / \mathrm{mL}$ ) or deficient $(<$ or $=20 \mathrm{ng} / \mathrm{mL}$ ). The lowest serum 25OHD level was observed in II-1's younger sister. Air pollution and a lack of exposure to sunlight may be the major factors responsible for the family's current vitamin D status. The serum sclerostin levels were approximately two times the ULN in the two patients, whereas no significantly decreased $\beta$-CTX levels were detected. In 2011, Frost (23) reported the sclerostin levels to increase in patients with high bone mass caused by the T253I mutation in LRP5. Sclerostin is an inhibitor of Wnt signaling that is secreted by osteoblasts and osteocytes (24). Although the antagonistic effects of sclerostin on Wnt signaling in LRP5 mutation-related high bone mass phenotypes have been reported (25), the mechanisms that produce elevated sclerostin levels and high bone mineral density in the LRP5 mutation and the Wnt signaling pathway remain to be elucidated.

In summary, screening for disease-causing mutations is important for the evaluation, clinical diagnosis, follow-up care and genetic counseling of patients with osteosclerosis. In this osteosclerotic family, the A242T mutation in LRP5 resulted in the high bone mass phenotype with an elongated mandible and torus palatinus. The mechanisms underlying the elevated serum sclerostin levels, the A242T mutation and the phenotypes of osteosclerosis should therefore be investigated in a further study.

The authors state that they have no Conflict of Interest (COI).

\section{Acknowledgement}

We thank the patients and their family members for their invaluable cooperation. Written consent for publication was obtained from the patients and their relatives.

This study was supported by the National Natural Science Foundation of China (NSFC) (nos. 30771019, 30800387, and 81070692); the Program of Shanghai Chief Scientist (project nos. 08XD1403000 and STCSM10DZ1950100); the Shanghai Science \& Technology Development Fund (project no. 11ZR 1427300); and the Shanghai Rising-Star program (11QA 1404900).

Chun Wang and Bao-Hong Zhang contributed equally to this paper.

\section{References}

1. Worth HM, Wollin DG. Hyperostosis corticalis generalisata congenita. J Can Assoc Radiol 17: 67-74, 1966.

2. Gorlin RJ, Glass L. Autosomal dominant osteosclerosis. Radiology 125: 547-548, 1977.

3. Van Wesenbeeck L, Cleiren E, Gram J, et al. Six novel missense mutations in the LDL receptor-related protein 5 (LRP5) gene in different conditions with an increased bone density. Am J Hum Genet 72: 763-771, 2003.

4. Boyden LM, Mao J, Belsky J, et al. High bone density due to a mutation in LDL-receptor-related protein 5. N Engl J Med 346: 1513-1521, 2002.

5. Ai M, Heeger S, Bartels CF, Schelling DK. Clinical and molecular findings in osteoporosis-pseudoglioma syndrome. Am J Hum Genet 77: 741-753, 2005.

6. Little RD, Carulli JP, Del Mastro RG, et al. A mutation in the LDL receptor-related protein 5 gene results in the autosomal dominant high-bone-mass trait. Am J Hum Genet 70: 11-19, 2002.

7. Gu JM, Zhang ZL, Zhang H, et al. Thirteen Chinese patients with sporadic Paget's disease of bone: clinical features, SQSTM1 mutation identification, and functional analysis. J Bone Miner Metab 30: 525-533, 2012.

8. Gao G, Zhang ZL, Zhang H, et al. Hip axis length changes in 10,554 males and females and the association with femoral neck fracture. J Clin Densitom 11: 360-366, 2008.

9. Richards JB, Rivadeneira F, Inouye M, et al. Bone mineral density, osteoporosis, and osteoporotic fractures: a genome-wide association study. Lancet 371: 1505-1512, 2008.

10. Hey PJ, Twells RC, Phillips MS, et al. Cloning of a novel member of the low-density lipoprotein receptor family. Gene 216: 103$111,1998$.

11. Jeon H, Meng W, Takagi J, Eck MJ, Springer TA, Blacklow SC. Implications for familial hypercholesterolemia from the structure of the LDL receptor YWTD-EGF domain pair. Nat Struct Biol 8 : 499-504, 2001.

12. de Vernejoul MC. Sclerosing bone disorders. Best Pract Res Clin Rheumatol 22: 71-83, 2008.

13. Janssens K, Van Hul W. Molecular genetics of too much bone. Hum Mol Genet 11: 2385-2393, 2002.

14. Kato M, Patel MS, Levasseur R, et al. Cbfa1-independent decrease in osteoblast proliferation, osteopenia, and persistent em- 
bryonic eye vascularization in mice deficient in Lrp5, a Wnt coreceptor. J Cell Biol 157: 303-314, 2002.

15. Ai M, Holmen SL, Van Hul W, Williams BO, Warman ML. Reduced affinity to and inhibition by DKK1 form a common mechanism by which high bone mass-associated missense mutations in LRP5 affect canonical Wnt signaling. Mol Cell Biol 25: 49464955, 2005.

16. Cohen MM Jr. The new bone biology: pathologic, molecular, and clinical correlates. Am J Med Genet A 140: 2646-2706, 2006.

17. Koay MA, Brown MA. Genetic disorders of the LRP5-Wnt signalling pathway affecting the skeleton. Trends Mol Med 11: 129137, 2005.

18. Kwee ML, Balemans W, Cleiren E, et al. An autosomal dominan high bone mass phenotype in association with craniosynostosis in an extended family is caused by an LRP5 missense mutation. J Bone Miner Res 20: 1254-1260, 2005.

19. Bhat BM, Allen KM, Liu W, et al. Structure-based mutation analysis shows the importance of LRP5 beta-propeller 1 in modulating Dkk1-mediated inhibition of Wnt signaling. Gene 391: 103-
112, 2007.

20. Beals RK. Endosteal hyperostosis. J Bone Joint Surg Am 58: 1172-1173, 1976.

21. Beals RK, McLoughlin SW, Teed RL, McDonald C. Dominant endosteal hyperostosis. Skeletal characteristics and review of the literature. J Bone Joint Surg Am 83-A: 1643-1649, 2001.

22. Scopelliti D, Orsini R, Ventucci E, Carratelli D. Van Buchem disease. Maxillofacial changes, diagnostic classification and general principles of treatment. Minerva Stomatol 48: 227-234, 1999.

23. Frost M, Andersen T, Gossiel F, et al. Levels of serotonin, sclerostin, bone turnover markers as well as bone density and microarchitecture in patients with high-bone-mass phenotype due to a mutation in Lrp5. J Bone Miner Res 26: 1721-1728, 2011.

24. Li X, Zhang Y, Kang H, et al. Sclerostin binds to LRP5/6 and antagonizes canonical Wnt signaling. J Biol Chem 280: 1988319887, 2005.

25. Balemans W, Piters E, Cleiren E, et al. The binding between sclerostin and LRP5 is altered by DKK1 and by high-bone mass LRP 5 mutations. Calcif Tissue Int 82: 445-453, 2008.

(C) 2013 The Japanese Society of Internal Medicine http://www.naika.or.jp/imonline/index.html 